\title{
Epidemiology of Injection Drug Use among Teenagers Infected with HIV and Hepatitis in the US
}

\author{
Adekunle Sanyaolu ${ }^{1 *}$, Chantel Zern ${ }^{2}$, Austin Fletcher ${ }^{2}$, Dorin Ghelmegeanu ${ }^{2}$, \\ Dmitry Roth ${ }^{2}$, Alvin Lapuz ${ }^{2}$, Michael Massengale ${ }^{2}$, Andel Higgins ${ }^{2}$ and \\ Charles Alaofin ${ }^{2}$ \\ ${ }^{1}$ Federal Ministry of Health Abuja, Nigeria \\ ${ }^{2}$ Saint James School of Medicine, Anguilla BWI, Nigeria
}

\section{Review Article}

Volume 5 Issue1

Received Date: January 26, 2021

Published Date: February 04, 2021

DOI: $10.23880 / \mathrm{jidtm}-16000145$

*Corresponding author: Dr. Adekunle Sanyaolu, Federal Ministry of Health Abuja, Nigeria, Email: sanyakunle@gmail.com

\section{Abstract}

The use of injection drugs amongst teenagers in the United States has been increasing over recent years and has been associated with various blood-borne infections. The morbidity and mortality are frequently analyzed with a focus on geographical and demographical makeup which has also been linked to the various types of injection drug use and might be more prevalent in one area than another. The analysis of social and economic factors has also been shown as factors in the increasing prevalence of injection drug use among United States teenagers. Evidence from various fields, such as sociology, psychology, anthropology, and epidemiology strongly suggest that the etiology of substance use and abuse is multifactorial, with genetics, psychological, and social factors collectively playing an influential role. This epidemiologic narrative review aimed to discuss blood-borne infections like HIV and Hepatitis amongst teenagers on injection drug use in the United States and elucidate further the role of the social and socioeconomic factors.

Keywords: Injection Drugs; Teenagers; United States; HIV; Hepatitis

\section{Introduction}

In the United States, the use of injection drug has been increasing over the past years, especidally among teenagers. Injected drugs are drugs that are introduced into the bloodstream using a needle and syringe [1]. Some of the injected drugs include methamphetamine, heroin, and ketamine. However, these drugs have various street names that are commonly used by the public. The type of drug injected varies depending on the geographic location and demographic makeup of the area [1].

The increase in the use of injection drugs can be attributable to various socioeconomic factors, such as stress, risky sexual behaviors, previous drug use, peer pressure, and drug use in the family. Even though there are many contributing factors, the use of injection drugs has also led to an increased risk for acquiring blood-borne diseases like HIV and Hepatitis [2]. Multiple other blood-borne infections can be acquired due to injection drug use.

Many researchers have attempted to analyze the use of injection drugs in teenagers, but little is known about the population prevalence of young injection drug users [2]. Nevertheless, researchers have shown that adolescent crack smokers, for instance, transition relatively quickly from a short period of exclusive crack smoking to a brief period of other non-injection drug use, and ultimately resort to injection drug use [3]. Hence, we carried out this epidemiologic narrative review on blood-borne infections like HIV and Hepatitis amongst teenagers on injection drug use in the United States and to elucidate further the role of the social and socioeconomic factors.

\section{Methodology}

A literature search for Injection drug use and bloodborne infections like HIV and Hepatitis was carried out. 
Articles were retrieved by performing searches using online electronic databases (Pub Med, Medline plus, Mendeley, Google Scholar, Research Gate, Global Health, and Scopus). Articles were streamlined to epidemiology, social and socioeconomic factors in the United States. Titles and abstracts of these results were reviewed and selected for inclusion based on relevancy to the research question. Overall, 44 articles and references were utilized for the study.

\section{Morbidity and Mortality Associated with Injection Drug Use}

Half of all new HIV diagnosis in the United States occurs in individuals younger than 25 years of age. $10 \%$ of new HIV cases worldwide are associated with injection drug use, also, 25 countries have reported greater than $20 \%$ prevalence of HIV occurring in injection drug users. Young intravenous drug users are at high risk of acquiring HIV and Hepatitis C. Young injection drug users are at higher risk compared to their older counterparts [2]. These findings are attributed to young injection drug users being less aware of the risks associated with injecting drugs and how to attenuate these risks [2]. They are also more likely to share needles and syringes. Young injection drug users also inject more frequently, engage in sexual activity with multiple partners, and often exchange sex for money and drugs [4].

The increasing prevalence of young intravenous drug users will likely increase the number of HIV/AIDS, hepatitis, hepatocellular carcinoma, and cirrhosis cases. Injection drug users are known to be at greater risk of contracting HIV, hepatitis B virus, and hepatitis C virus [2]. Younger age and shorter-term drug use are risk factors for HIV infection [5]. Young age has been shown in numerous cross-sectional studies to be associated with HIV infection and high-risk injection practices [2], High-risk sexual practices such as prostitution, inconsistent condom use, multiple sex partners, and homosexual sex are also independently associated with HIV infection [4]. Injection practices including injecting cocaine and speed balling are also associated with HIV infection. Studies have also shown that a strong association exists between injection drug use and risky sexual behavior at a young age [2].

HIV drug users also commonly have associated psychiatric disorders [5]. Factors related to positive screening for the mental disorder include several HIVrelated symptoms, illicit drug use, drug dependence, heavy alcohol use, and being unemployed or disabled [5]. Factors associated with drug addiction in this population include having many HIV symptoms present, younger age, being homosexual, frequent alcohol use, and psychiatric disorder [5]. The prevalence of drug use and drug dependence among people with HIV disease is high, due to the association between injection drug use and HIV transmission [5]. Drug use is shown as an increasing trend for the primary transmission route of infection and stands as the cause of one-third of cases of acquired immunodeficiency syndrome (AIDS) [1,5].

Research suggests the rate of psychiatric disorders and substance abuse vary depending on the population studied [5]. Depression in HIV-infected populations ranges from $22 \%$ to $32 \%, 2$ to 3 times higher than the prevalence of depression in general populations [5]. The rates of disorders among homosexual men studies are high although rates of psychiatric disorders have also been found in the general population studies of homosexual men [5]. Studies that have also included comparisons from the population as those who are HIV positive have not found differences in the prevalence of psychiatric disorders [5].

HIV-infected patients who concomitantly suffer from mental disorders had an impaired quality of life [5]. This adversely affects their ability to manage their HIV and seek out health care services. This ultimately affects the course of the disease, compromising compliance with the medical treatment plan and prescribed medications [5]. Psychiatric disorders also increase the likelihood that these individuals will engage in unsafe sex practices and needle sharing behavior [5]. This ultimately leads to the spread of HIV to other drug users. The social burden of this problem is substantial and poses greater reaching health care concerns [5]. Infection with HIV and the possibility of having associated psychiatric disorders and drug dependence must be taken into consideration by health care providers when proving care for their patients [5].

Psychiatric disorders including depression, anxiety, and severe mental illness increase the risk of acquiring HIV in injection drug users [5]. Also associated is reduced compliance with highly active antiretroviral therapy [5]. Non-compliance with medication in general in patients with schizophrenia, for example, is a high as $50 \%$, demonstrating the challenges patients with HIV and mental illness have. Financial barriers are also a factor in compliance with medications. Many injection drug users may not have adequate health insurance or may choose to purchase drugs over medication to manage their conditions. This is significant as both HIV and many psychiatric disorders require pharmacological management [5].

Injection drug use is highly associated with the hepatitis C virus [6]. 35,000 people are infected with the hepatitis $C$ virus in the United States annually. $60 \%$ of these cases occur in injection drug users [6]. The largest portions of hepatitis $\mathrm{C}$ infected individuals in the United States are injection drug users. Most new infections continue to occur in injection 
drug users, greater than any other risk factor associated with disease [7]. Hepatitis $\mathrm{C}$ antibody prevalence in injection drug users is estimated to be around $80-90 \%$. Incidence rates of hepatitis $\mathrm{C}$ in this population continue to rise annually, current estimates believe the incidence rate to be around $10 \%-20 \%[6]$.

Progression of hepatitis $C$ is linked to clinical outcomes including cirrhosis, liver cancer, end-stage liver disease, and death [6]. Although possible disease outcomes are known, little research exists on the outcome of hepatitis $C$ in injection drug users. Future studies should consider the significance of this issue, as injection drug users have different risk factors than the general population [6]. These include frequency of exposure, nutritional status, co-infections with various infectious agents, and other factors that may affect the natural history of the disease. Studies also need to be conducted on the appropriate treatment for this population and risk reduction strategies to reduce the transmission of hepatitis $\mathrm{C}$ among injection drug users [6].

\section{The Incidence and Prevalence of Injection Drug Use in the US}

Research indicates that young injection drug users (IDUs) widely differ in their risk behavior from individuals in an older demographic, data has shown that younger injection drug users have a very high risk of HIV, HCV, STIs, and drug overdose [7]. Young injection drug users have a decreased awareness of the dangers of injecting drugs and how to reduce their risk, and more likely to share syringes and drug paraphernalia needed for injection. They inject drugs more frequently, have multiple sexual partners, and exchange sex for money or drugs more often [8].

Since young IDUs are at risk for acquiring blood-borne diseases like HIV and Hepatitis $\mathrm{C}$, we must ascertain a general baseline of data to manage better the secondary effects of injection drug use, however, little is known about the population prevalence of young IDUs. Data on the prevalence of young IDUs in US geographic areas are rare [8]. The National Survey on Drug Use and Health (NSDUH) provides annual estimates of numbers of young IDUs in the US, but these data are not suitable to measure change as (a) data are derived from a household survey with well-known sampling and self-report limitations; and (b) NSDUH acknowledge the data are not suitable for longitudinal analyses, given changes in data collection methods over time [8].

One comprehensive study estimated annual population prevalence rates of young IDUs (aged 15-29) per 10,000 in 95 large U.S. metropolitan statistical areas (MSAs) from 1992 to 2002 assessed the validity of these estimates; and, explored whether injection drug use among youth in these
MSAs began to rise after HAART was discovered [2]. A linear mixed model (LMM) estimated the annual population prevalence of young IDUs in each MSA and described trends therein. The population prevalence of IDUs among youth across 95 MSAs increased from 1996 (mean $=95.64)$ to 2002 ( mean $=115.59)$. Additional analyses of 2002 [2].

These data suggest that the prevalence of IDUs among adolescents and young adults increased between 1995 and 2002. Further analyses of the proportions of IDUs entering treatment or receiving HIV counseling and testing who were young suggest that this trend may have continued through 2005. In another study that extended beyond 2005 and which monitored the prevalence of injection drug use among young adults aged 21-30 in the US, the data regarding gender differences was of a greater focus [8]. In the nineyear (2004-2012) combined sample of young adults aged $21-30,1.6 \%$ report having ever used any drug by injection without medical supervision [8]. There is a fair-sized gender difference $-2.4 \%$ of males, and $0.9 \%$ of females indicate such behavior [8]. The percentage saying, they injected on 40 , or more occasions are $0.5 \%$ overall $-0.6 \%$ for males and $0.4 \%$ for females [8]. Therefore, a relatively limited segment of respondents has ever used an illicit drug by injection about 1 in every 60; a smaller proportion [8]. About 1 in every 200 respondents reports an extended pattern of use as indicated by use on 40 or more occasions. The proportions of young adults who have injected drugs during the past 12 months without medical supervision are considerably smaller: $0.5 \%$ overall-1 in every 200 respondents-including $0.8 \%$ of males and $0.3 \%$ of females (an extremely significant gender difference) [8]. The proportions using 40 or more times in the past 12 months are $0.2 \%$ overall $-0.2 \%$ for males, and $0.1 \%$ for females. It is interesting to note that the proportional difference between the genders is larger for having injected drugs in their lifetime (2.4\% of males versus $0.9 \%$ of females) than it is for having ever shared needles $(0.5 \%$ of males versus $0.4 \%$ of females), suggesting that females' injectors are more at risk of needle sharing [8].

The disparity in disease burden among those who inject drugs compared to their respective population size has been difficult to quantify. Although the Centers for Disease Control and Prevention (CDC) routinely uses demographic data from the Census Bureau to calculate disease rates by selected demographic categories (e.g., sex, race/ethnicity, and age at diagnosis), no census data are available for the number of PWID in the U.S. and rate calculations require this number for the denominator [9].

\section{Types of Drugs of Abuse and Statistics}

Research by Lankenau [10] suggested that the most injected drugs that are used among teenagers in the United 
States are heroin, methamphetamine, cocaine, and ketamine. The percentages of young IDUs who have initiated with four of the common drug types are as follows: heroin (48.6\%), methamphetamine $(20.3 \%)$, ketamine $(17.1 \%)$, and cocaine (14\%) [10]. Regardless of the percentages, the various drug types that were injected at initial events, such as heroin, methamphetamine, cocaine, or ketamine, have often resulted in limited findings and for the most part, received less research attention. For instance, when young IDUs were compared, who started with heroin first versus methamphetamine, no differences were yielded by the rationale/motive for injecting, the percentage of who planned their first injection, or the proportion of who first committed self-injection [10]. Hence, qualitative analyses by Lankenau [10] have minimally shown rationales for initiation of injections that differed among the four common drug types.
The study of Lankenau [10] as shown in (Table 1) reported the sample demographics and the different health histories by drug injected at initial events. Overall, the mean age at initiation was 16.5 years old with subjects being primarily white, male, and heterosexual. A majority of these IDUs had histories of mental health care, HIV/HCV testing, and most were unemployed. No significant differences were yielded among the variables by drug type. However, significant differences were reported between initiates of ketamine and other drugs on six other variables. For instance, age at initiation of injection was different by drug type: initiates of ketamine started their injection career at 18.8 years old, whereas cocaine initiates started theirs at 16.4 years of age, methamphetamine initiates at 16.2 years old, and heroin initiates at 15.9 years old $(p<0.001)$.

\begin{tabular}{|c|c|c|c|c|c|}
\hline & Total & Heroin (1) & Meth (2) & Ketamine (3) & Cocaine (4) \\
\hline & $\mathrm{n}=\mathbf{2 2 2}$ & $n=108$ & $n=45$ & $\mathrm{n}=38$ & $n=31$ \\
\hline Mean Age at Enrollment & 22.3 & 22.4 & 22.5 & 21.6 & 22.3 \\
\hline Mean Age at Initiation & 16.5 & 15.9 & 16.2 & 18.8 & 16.4 \\
\hline Male Gender & $69.80 \%$ & $69.40 \%$ & $68.90 \%$ & $68.40 \%$ & $74.20 \%$ \\
\hline \multicolumn{6}{|l|}{ Race and Ethnicity } \\
\hline White/Caucasian & $74.80 \%$ & $79.60 \%$ & $75.60 \%$ & $71.10 \%$ & $61.30 \%$ \\
\hline Black/African American & $0.50 \%$ & $0 \%$ & $2.20 \%$ & $0 \%$ & $0 \%$ \\
\hline Hispanic/Latino & $5.90 \%$ & $4.60 \%$ & $6.70 \%$ & $2.60 \%$ & $12.90 \%$ \\
\hline Asian or Pacific Islander & $0.50 \%$ & $0.90 \%$ & $0 \%$ & $0 \%$ & $0 \%$ \\
\hline Native American & $0.90 \%$ & $1.90 \%$ & $0 \%$ & $0 \%$ & $0 \%$ \\
\hline Multiracial Background & $17.60 \%$ & $13.00 \%$ & $15.60 \%$ & $26.30 \%$ & $25.80 \%$ \\
\hline \multicolumn{6}{|l|}{ Sexual Identity } \\
\hline Heterosexual & $78.40 \%$ & $79.60 \%$ & $84.40 \%$ & $76.30 \%$ & $67.70 \%$ \\
\hline Gay/Lesbian & $1.80 \%$ & $2.80 \%$ & $0 \%$ & $2.60 \%$ & $0 \%$ \\
\hline Bisexual & $16.70 \%$ & $16.70 \%$ & $11.10 \%$ & $13.20 \%$ & $29.00 \%$ \\
\hline Other/Undecided & $4.60 \%$ & $1.90 \%$ & $6.70 \%$ & $7.90 \%$ & $6.50 \%$ \\
\hline High School Graduate or GED & $61.30 \%$ & $61.10 \%$ & $44.40 \%$ & $76.30 \%$ & $67.70 \%$ \\
\hline Homeless & $79.90 \%$ & $78.60 \%$ & $87.20 \%$ & $62.10 \%$ & $92.60 \%$ \\
\hline Homeless Traveler & $60.90 \%$ & $64.30 \%$ & $64.10 \%$ & $37.90 \%$ & $70.40 \%$ \\
\hline Employed Full or Part Time & $27.50 \%$ & $33.30 \%$ & $17.80 \%$ & $34.20 \%$ & $12.90 \%$ \\
\hline History of Drug Treatment & $53.20 \%$ & $60.20 \%$ & $57.80 \%$ & $31.60 \%$ & $48.40 \%$ \\
\hline History of Mental Health Care & $73.90 \%$ & $70.40 \%$ & $80.00 \%$ & $71.10 \%$ & $80.60 \%$ \\
\hline Ever arrested & $91.90 \%$ & $92.60 \%$ & $97.80 \%$ & $81.60 \%$ & $93.50 \%$ \\
\hline Ever in Jail & $86.90 \%$ & $88.90 \%$ & $95.60 \%$ & $73.70 \%$ & $83.90 \%$ \\
\hline Ever in Prison & $15.30 \%$ & $18.50 \%$ & $15.60 \%$ & $5.30 \%$ & $16.10 \%$ \\
\hline Tested for HIV & $91.90 \%$ & $92.60 \%$ & $93.30 \%$ & $84.20 \%$ & $96.80 \%$ \\
\hline HIV Positive & $0.50 \%$ & $0.90 \%$ & $0 \%$ & $0 \%$ & $0 \%$ \\
\hline Tested for HCV & $85.60 \%$ & $87.00 \%$ & $88.90 \%$ & $76.30 \%$ & $87.10 \%$ \\
\hline HCV Positive & $20.30 \%$ & $23.10 \%$ & $28.90 \%$ & $5.30 \%$ & $16.10 \%$ \\
\hline
\end{tabular}

Table 1: Demographic Characteristics of Initiates by Drug Type $(\mathrm{N}=222)^{10}$. 
Correlations between drug types and certain practices, detrimental health outcomes, and risk behaviors have been reported in various other studies that did not solely concentrate on injection initiation. For instance, IDUs of cocaine have been shown to inject with riskier social networks [11]. Injection of crack cocaine carries risks for venous damage because its usage often involves the use of lemon juice, ascorbic acid, or vinegar [12]. With heroin, it is often associated with varying HIV risks that may be dependent on its drug form such as tar or powder form [13]. Injection of methamphetamine has been correlated with increased sexual risk-taking, most specifically among men who have sex with other men [10]. Ketamine is often associated with risks attributable to the use of multi-dose pharmaceutical vials, which are susceptible during the events of ketamine injections [14].

\section{The Social Epidemiology of Injection Drug Use}

The study of the social epidemiology of substance use, a field that considers the social factors that shape the population distribution of substance use behavior, is critical because epidemiology is inherently social, people are biologic and social organisms with few behaviors unmediated by social context [15]. Tobacco, alcohol, and illicit substance use continue to result in substantial morbidity and mortality and significant societal economic costs despite considerable efforts to prevent and minimize the use of such materials [15]. Evidence from various fields, such as sociology, psychology, anthropology, and epidemiology strongly suggest that the etiology of substance use and abuse is multifactorial, with genetics, psychological, and social factors collectively playing a substantial role [15].

Consequences of illicit substance abuse include, among others, morbidity and mortality, job loss, and criminal behavior related to the acquisition and sale of illegal drugs, with economic costs of addiction estimated to be a total of $\$ 400$ billion per year in the United States in 1999 [16]. Various risk behaviors are associated with substance use, and individuals often have symptoms of dependence (e.g., reducing activities due to use of the material) and abuse (e.g., driving a car while intoxicated, getting into trouble at their place of employment). In 2002, 4.7\% reported driving under the influence of an illicit drug and 14.2\% under that of alcohol in the past year. Social network and familial characteristics, the latter to a lesser extent, are the primary social factors identified in the initiation of heroin or cocaine use [10]. Social network characteristics that have been associated with the initiation of injection drug use include having partners who injected drugs and having a high-risk social network [17]. A cross-sectional study of injection drug users during the first 3 weeks of their injection drug use demonstrated that adverse family conditions were associated with a greater likelihood of initiating injection drug use [17]. This study also identified individual social circumstances as determinants of initiation of injection, homelessness, and unemployment [17].

In a case-control study among African American heroin users, lower familial socioeconomic status was associated with younger age at initiation of heroin use among persons born in the United States but not among migrants, suggesting both that familial characteristics and cultural determinants (migration) play key roles [18]. In contrast to these findings, a study of Swedish conscripts found that lower familial socioeconomic status was associated with a lower likelihood of being offered a drug [18] and a cross-sectional study of 262 California adolescents found no consistent relationship between family socioeconomic status and initiation of substance use [19]. Several studies of the initiation of multiple drugs have assessed familial, social characteristics as potential determinants of substance initiation, also with conflicting results. Many other studies, however, have consistently associated disruption of family structure with the initiation of adolescent drug use [2027]. Social network characteristics, such as involvement in drug-using social networks, are also consistently associated with increased initiation of use of multiple substances [16]. Participation in religious activities, possibly a proxy for involvement with non-drug-using social networks, is associated with a lower likelihood of illicit drug initiation in one study [28]. In a study of 1,911 adolescents in South Carolina public schools, interactions were found between gender and family structure, with boys who did not have close family relationships being most likely to start using substances [27]. The role of race/ethnicity in substance use initiation has only been the topic of very few studies [28]. Educational attainment was a more significant predictor of initiation of substance use among Whites than among other races according to a study conducted on 778 students from Seattle, Washington elementary schools [29]. A prospective cohort study of 1,416 students found that neighborhood disadvantage was associated with the initiation of drug use, with a more pronounced effect for illicit (cocaine) than for licit (tobacco, alcohol) drugs [30].

Ultimately, while adverse family conditions during childhood appear to be an important social determinant of illicit drug use, there is no apparent relation between family socioeconomic status and initiation of illegal drug use [16]. Characteristics of social networks such as drug use in the peer network are prominently studied as social determinants of initiating illicit drug use. Few studies have been designed to test competing familial or peer network influences [16]. Individual characteristics, including gender and race, likely modify the association of adverse family conditions and social network characteristics with the initiation of drug use 
[16]. Parental illicit drug use also has been associated with illegal drug use by adults in some studies [26,31].

The use of illegal drugs by members of one's social networks is associated with a greater likelihood of drug use [32-34]. Adverse childhood family conditions have been associated with a greater likelihood of multiple drug use [35]. In a study of 1,101 White and African American adults from Detroit, Michigan, an association was found between neighborhood disadvantage and drug use after the authors controlled for individual social and psychological resources [36].

Social factors associated with cessation of illicit drug use are like that of cigarette and alcohol cessation [16]. In a prospective cohort study of 706 marijuana users, higher education was associated with the cessation of marijuana use [37]. Becoming a parent for the first time has been associated with cessation of marijuana use and having children has been associated with entry into methadone maintenance by injection drug-using women $[37,38]$. Although socioeconomic factors are not consistently associated with cessation of illicit drug use, homelessness is associated with a lower likelihood of seeking treatment for drug use and employment with an increased probability of seeking treatment [38]. Importantly, several studies have found social supports and increased family responsibility to be associated with cessation of illicit drug use [16]. According to one prospective cohort study of 439 daily heroin users admitted to three communitybased methadone maintenance clinics, reduction of family conflict, which was likely indicative of strengthening social supports, was associated with a decreased likelihood of drug injection [39]. Social supports and social network norms not supportive of illicit drug use have been associated with cessation and sustained abstinence $[40,41]$. A smaller proportion of drug users in one's social networks are a significant predictor of cessation; therefore, family and social network norms are the factors primarily associated with cessation of illicit drug use and sustained abstinence [42]. Future social epidemiologic research should aim to study not only how social factors may influence substance use in isolation, but also how such factors modify relations between endogenous variables and substance use behavior [16].

\section{Geographic Epidemiology of Injectable Drug Use Amongst Teenagers in the United States}

The geographical pattern of the use of injectable drugs among teenagers in the United States is of varying degrees depending on what type of drug is in question and the proximity of the cities or towns to international borders and the demography of the inhabitants of that region of the country. It has been demonstrated that drug-related crimes and transactions are more common in states like Texas, Arizona, New Mexico, and California just to mention a few due to their accessibility to the borders, most of these areas were designated high-intensity drug trafficking areas[HIDTA] by the Anti-Drug Abuse Act of 1988 and the ONDCP Reauthorization Act of 1998 which help provides additional federal resources to those areas to help eliminate or reduce drug trafficking and its harmful consequences [43].

\section{Conclusion}

Intravenous drug use among adolescents and teens has been steadily rising over the years. This predisposes many teens to share needles which make blood-borne diseases such as HIV, Hepatitis B, and Hepatitis C much more likely to be acquired. It has been shown that intravenous drug use primarily caused approximately one-third of HIV/AIDS cases. Among the users, studies showed that the initiation and cessation were a result of multiple factors.

The studies that were done suggested that family and peers played a huge role in the initiation of intravenous drug use. Single-parent families, as well as families that were not close, are more likely to initiate IV substance abuse in teens and adolescents possibly due to less parental monitoring. Also, the general acceptance of drug use or family members using drugs was more likely to initiate the behavior. Studies also showed that negative incidences in a child could have influenced it as well. In multiple studies, it was shown that peers in an individual's social network could greatly influence the initiation of the drug use, factors such as peer acceptance, usage, and peer pressures. Smaller factors such as unemployment and depression were seen to correlate with the behavior. On a few studies, it was seen that social, economic status had no significance in the initiation while religious household showed teens were one-fifth less likely to start.

The studies that dealt with adolescent intravenous drug relapse showed a few correlating factors. It was seen that positive family events and fewer friends in an individual's social network who use IV drugs were less likely to relapse after discontinuing the drugs. However, individuals who were living with IV drug users or who had more drug-using peers, in general, had a less likely probability of cessation.

\section{References}

1. AIDS.gov (2014) Substance abuse/use.

2. Chatterjee S, Tempalski B, Pouget ER, Cooper HL, Cleland 
CM, et al. (2011) Changes in the prevalence of injection drug use among adolescents and young adults in large US metropolitan areas. AIDS Behav 15(7): 1570-1578.

3. Fuller CM, Vlahov D, Arria AM, Ompad DC, Garfein R, et al. (2001) Factors associated with adolescent initiation of injection drug use. Public Health Rep 116(1): 136-145.

4. Fuller CM, Vlahov D, Latkin CA, Ompad DC, Celentano DD, et al. (2003) Social circumstances of initiation of injection drug use and early shooting gallery attendance: implications for HIV intervention among adolescent and young adult injection drug users. JAIDS 32(1): 86-93.

5. Bing EG, Burnam MA, Longshore D, Fleishman JA, Sherbourne CD, et al. (2001) Psychiatric disorders, and drug use among human immunodeficiency virusinfected adults in the United States. Arch Gen Psychiatry 58(8): 721-728.

6. Edlin BR (2002) Prevention and treatment of hepatitis C in injection drug users. Hepatology 36(5S1): S210-219.

7. Miller CL, Spittal PM, LaLiberte N, Li K, Tyndall MW, et al. (2002) Females experiencing sexual and drug vulnerabilities are at elevated risk for HIV infection among youth who use injection drugs. J Acquir Immune Defic Syndr 30(3): 335-341.

8. Thorpe LE, Ouellet LJ, Levy JR, Williams IT, Monterroso ER (2000) Hepatitis C virus infection: prevalence, risk factors, and prevention opportunities among young injection drug users in Chicago, 1997-1999. J Infect Dis 182(6): 1588-1594.

9. Lansky A, Finlayson T, Johnson C, Holtzman D, Wejnert $C$, et al. (2014) Estimating the number of persons who inject drugs in the United States by meta-analysis to calculate national rates of HIV and hepatitis $\mathrm{C}$ virus infections. PloS one 9(5): e97596.

10. Lankenau SE, Wagner KD, Bloom JJ, Sanders B, Hathazi D, et al. (2010) The first injection event: differences among heroin, methamphetamine, cocaine, and ketamine initiates. J Drug Issues 40(2): 241-262.

11. De P, Cox J, Boivin JF, Platt RW, Jolly AM (2007) Rethinking approaches to risk reduction for injection drug users: Differences in drug type affect risk for HIV and hepatitis C virus infection through drug-injecting networks. J Acquir Immune Defic Syndr 46(3): 355-361.

12. Lankenau SE, Clatts MC, Goldsamt LA, Welle D (2004) Crack cocaine injection practices and HIV risk: findings from New York and Bridgeport. J Drug Issues 34(2): 319332.
13. Clatts MC, Heimer R, Abdala N, Goldsamt LA, Sotheran JL, et al. (1999) HIV-1 transmission in injection paraphernalia: heating drug solutions may inactivate HIV-1. Journal of acquired immune deficiency syndromes 22(2): 194-199.

14. Lankenau SE (2006) On ketamine: In and out of the Khole Drugs, clubs, and young people: Sociological and public health perspectives. Aldershot Ashgate Publishers, United Kingdom, pp: 77-87.

15. Galea S, Nandi A, Vlahov D (2004) The social epidemiology of substance use. Epidemiol Rev 26(1):36-52.

16. McGinnis JM, Foege WH. (1999) Mortality, and morbidity attributable to use of addictive substances in the United States. Proc Assoc Am Physicians 111(2): 109-118.

17. Neaigus A, Miller M, Friedman SR, Hagen DL, Sifaneck SJ, et al. (2001) Potential risk factors for the transition to injecting among non-injecting heroin users: a comparison of former injectors and never injectors. Addiction 96(6): 847-860.

18. Kleinman PH (1978) Onset of addiction: A first attempt at prediction. Int J Addict 13(8): 1217-1235.

19. Fawzy FI, Coombs RH, Simon JM, Bowman-Terrell M (1987) Family composition, socioeconomic status, and adolescent substance use. Addictive behaviors 12(1): 79-83.

20. Boys A, Farrell M, Bebbington P, Brugha T, Coid J, et al. (2002) Drug use and initiation in prison: results from a national prison survey in England and Wales. Addiction 97(12): 1551-1560.

21. De Wit ML, Embree BG, De Wit D (1999) Determinants of the risk and timing of alcohol and illicit drug use onset among natives and non-natives: Similarities and differences in family attachment processes. Soc Biol 46(1-2): 100-121.

22. Dube SR, Felitti VJ, Dong M, Chapman DP, Giles WH, et al. (2003) Childhood abuse, neglect, and household dysfunction and the risk of illicit drug use: the adverse childhood experiences study. Pediatrics 111(3): 564572.

23. Guo JI, Hill KG, Hawkins JD, Catalano RF, Abbott RD (2002) A developmental analysis of sociodemographic, family, and peer effects on adolescent illicit drug initiation. J Am Acad Child Adolesc Psychiatry 41(7): 838-845.

24. Sobeck J, Abbey A, Agius E, Clinton M, Harrison K (2000) Predicting early adolescent substance use: Do risk factors differ depending on age of onset? J Subst Abuse 
11(1): 89-102.

25. Von Sydow K, Lieb R, Pfister H, Hofler M, Wittchen HU (2002) What predicts incident use of cannabis and progression to abuse and dependence? A 4-year prospective examination of risk factors in a community sample of adolescents and young adults. Drug Alcohol Depend 68(1): 49-64.

26. Wallace Jr JM, Forman TA, Guthrie BJ, Bachman JG, O'Malley PM, et al. (1999) The epidemiology of alcohol, tobacco, and other drug use among black youth. J Stud Alcohol 60(6): 800-809.

27. Williams JG, Smith JP (1993) Alcohol and other drug use among adolescents: Family and peer influences. Journal of Substance Abuse 5(3): 289-294.

28. Johanson CE, Duffy FF, Anthony JC (1996) Associations between drug use and behavioral repertoire in urban youths 1. Addiction 91(4): 523-534.

29. Wells EA, Morrison DM, Gillmore MR, Catalano RF, Iritani B, et al. (1992) Race differences in antisocial behaviors and attitudes and early initiation of substance use. J Drug Educ 22(2): 115-130.

30. Crum RM, Lillie-Blanton M, Anthony JC (1996) Neighborhood environment and opportunity to use cocaine and other drugs in late childhood and early adolescence. Drug Alcohol Depend 43(3): 155-161.

31. Lindenberg CS, Strickland O, Solorzano R, Galvis C, Dreher M, et al. (1999) Correlates of alcohol and drug use among low-income Hispanic immigrant childbearing women living in the USA. Int J Nurs Stud 36(1): 3-11.

32. Madianos MG, Gefou-Madianou D, Richardson C, Stefanis CN (1995) Factors affecting illicit and licit drug use among adolescents and young adults in Greece. Acta Psychiatr Scand 91(4): 258-264.

33. Newcomb MD, Bentler PM (1986) Cocaine use among young adults. Adv Alcohol Subst Abuse 6(2): 73-96.

34. Schroeder JR, Latkin CA, Hoover DR, Curry AD, Knowlton $A R$, et al. (2001) Illicit drug use in one's social network and in one's neighborhood predicts individual heroin and cocaine use. Ann Epidemiol 11(6): 389-394.

35. Tam TW, Weisner C, Mertens J (2000) Demographic Characteristics, Life Context, and Patterns of Substance Use Among Alcohol-Dependent Treatment Clients in a Health Maintenance Organization. Alcoholism: Clinical and Experimental Research 24(12): 1803-1810.

36. Boardman JD, Finch BK, Ellison CG, Williams DR, Jackson JS (2001) Neighborhood disadvantage, stress, and drug use among adults. Journal of health and social behavior 42(2): 151-165.

37. Chen K, Kandel DB (1998) Predictors of cessation of marijuana use: an event history analysis. Drug Alcohol Depend 50(2): 109-121.

38. Lundgren LM, Schilling RF, Fitzgerald T, Davis K, Amodeo M (2003) Parental status of women injection drug users and entry to methadone maintenance. Subst Use Misuse 38(8): 1109-1131.

39. Knight DK, Simpson DD (1996) Influences of family and friends on client progress during drug abuse treatment. Journal of Substance Abuse 8(4): 417-429.

40. Havassy BE, Wasserman DA, Hall SM (1995) Social relationships, and abstinence from cocaine in an American treatment sample. Addiction 90(5): 699-710.

41. Kandel DB, Raveis VH (1989) Cessation of illicit drug use in young adulthood. Arch Gen Psychiatry 46(2): 109116.

42. Latkin CA, Knowlton AR, Hoover D, Mandell W (1999) Drug network characteristics as a predictor of cessation of drug use among adult injection drug users: a prospective study. The American journal of drug and alcohol abuse 25(3): 463-473.

43. Center for Behavioral Health Statistics and Quality (2016) Key substance use and mental health indicators in the United States: Results from the 2015 National Survey on Drug Use and Health (HHS Publication No. SMA 16-4984, NSDUH Series H-51). 